\title{
In situ electrical testing of device-relevant nanocontacts in the transmission electron microscope
}

\author{
Daan Hein Alsem ${ }^{1}$, Siddharth Sood ${ }^{1}$, Norman Salmon ${ }^{1}$, and Tevis D. B. Jacobs ${ }^{2}$ \\ 1. Hummingbird Scientific, Lacey WA, USA. \\ 2. University of Pittsburgh, Department of Mechanical Engineering and Materials Science, Pittsburgh \\ PA, USA.
}

In situ transmission electron microscopy (TEM) experiments are finding increased use to explore the relationships between material processing methods, microstructure and functional properties. Although the benefit of direct observation of the material response to applied stimuli at high magnification has long been recognized, the development of new tools with higher precise and more versatility has enabled much more widespread use of the TEM for in situ characterization. In this study we show initial results obtained with a new in situ TEM electrical characterization tool that allows a movable probe, to make site-specific electrical contact measurements to study device-related nanoscale electrical contacts (Fig. 1).

The electrical properties of nanoscale contacts are of direct importance in small-scale devices - including probe-based microscopies (e.g. [1]) and nanomanufacturing techniques (e.g., [2]) as well as micro-/nanoelectromechanical systems (M/NEMS) (e.g.[3]). In many cases, electrical transport properties through the contact determine functionality of the device, yet the behavior of the contact conductance is multi-faceted and not easily characterized. There has been extensive characterization of the electrical properties of ultrasmall contacts using mechanically controllable break junctions and scanning probe techniques [4]. However, in these techniques the shape, size, and structure of the opposing bodies are typically unknown, as is the geometry of their contact. Thus, confounding factors such as the presence of oxide films and contaminants; the evolving shapes of the bodies due to inelastic deformation; and inaccurate estimation of contact sizes can cause uncertainty in experimental measurements of contact properties. In situ TEM measurements of electrical contacts can overcome these limitations. While investigations have been performed using in situ electrical measurements inside a TEM before - including on single-atom-width nanobridges in gold [5] - these methods typically require specially prepared contacts and a limited range of materials and geometries. The flexibility of the present in situ tool enables simple, repeatable and accurate probe positioning, high-resolution imaging, and accommodates a wide range of nanoscale contact samples with a removable holder tip.

Here two contact configurations that are common to conductive scanning probe microscopy were recreated in situ in the TEM. Namely, a W substrate was contacted by a sharp nanoscale tip that is composed either of Pt/Ir or of doped Si. We demonstrate that current-voltage sweeps can be performed with real-time imaging of the nanoscale bodies as well as their contact geometry. As shown in Fig. 2(a), the metal/metal contact is ohmic and the computed resistance is 729 ohms. By contrast, the metal/semiconductor interface (Fig. 2(b)) has a highly asymmetrical IV curve, displaying Schottky-like behavior - as is commonly seen in conductive probe microscopy with doped-silicon tips [6].

As an example of the benefit of in situ imaging of real-world electrical contacts, we can compute the contact resistivity of the metal/metal contact. The direct imaging of the geometry enables the measurement of an apparent contact radius of $9.8 \mathrm{~nm}$. The resistivity can be calculated using the classical (Maxwell), ballistic (Sharvin), or intermediate (Knudsen) limits [7]. The mean free path in a metal can be estimated 
from the Fermi velocity and the bulk conductivity [8], and was computed for $\mathrm{W}$ to be $\sim 15 \mathrm{~nm}$. Because this is on the order of the contact radius, the intermediate limit is appropriate, leading to a calculation of $\rho_{\text {Knudsen }}=620 \mu \Omega-\mathrm{cm}$. By having a direct measure of the apparent contact area - obviating the reliance on continuum contact models - we can compute resistivity directly. It should be noted that this value is much larger than the bulk resistivity of $\mathrm{W}$ which is $4.82 \mu \Omega-\mathrm{cm}$ [9]. This is likely attributable to the presence of insulating surface films (such as oxide or contamination) and/or to the fact that the true contact area is much smaller than the apparent contact area due to surface roughness.

\section{References:}

[1],JY Park et al, Materials today, 38 (2010), p. 38.

[2] C Cen et al, Nature Materials, 7 (2008), p.298

[3] OY Loh, HD Espinosa, Nature Nanotechnology, 7 (2012), p.283

[4] N Agrait, AL, Yeyati, JM van Ruitenbeek, Physics Reports, 377 (2003), p.81

[5] H Ohnishi, Y Kondo, K Takayanagi, Nature 395 (1998),p.780

[6] MA Lantz, SJ O’Shea, ME Welland, Review of scientific instruments 69 (1998), p.1757

[7] Wiesendanger, Scanning Probe Microscopy and Spectroscopy, Cambridge U. Press (1994)

[8] Ashcroft \& Mermin, Solid State Physics, Brooks Cole (1976)

[9] WM Haynes, ed. CRC handbook of chemistry and physics, CRC press (2014)

[10] T.D.B.J. acknowledges support from National Science Foundation under award

No. \#CMMI-1536800.

(a)

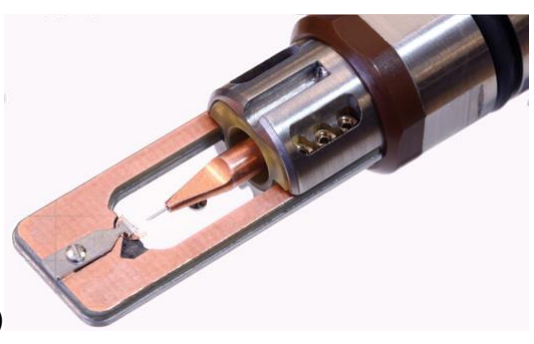

(b)

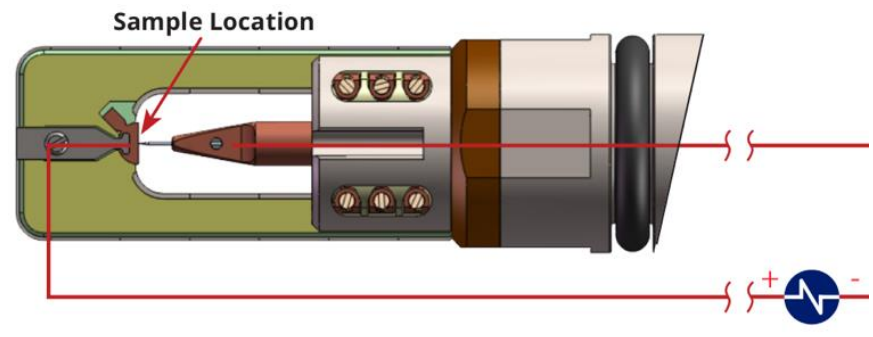

Figure 1. In situ nano-manipulating biasing TEM holder (a) holder tip; (b) schematic of the sample/probe and wiring schematic for in situ TEM electrical experiments
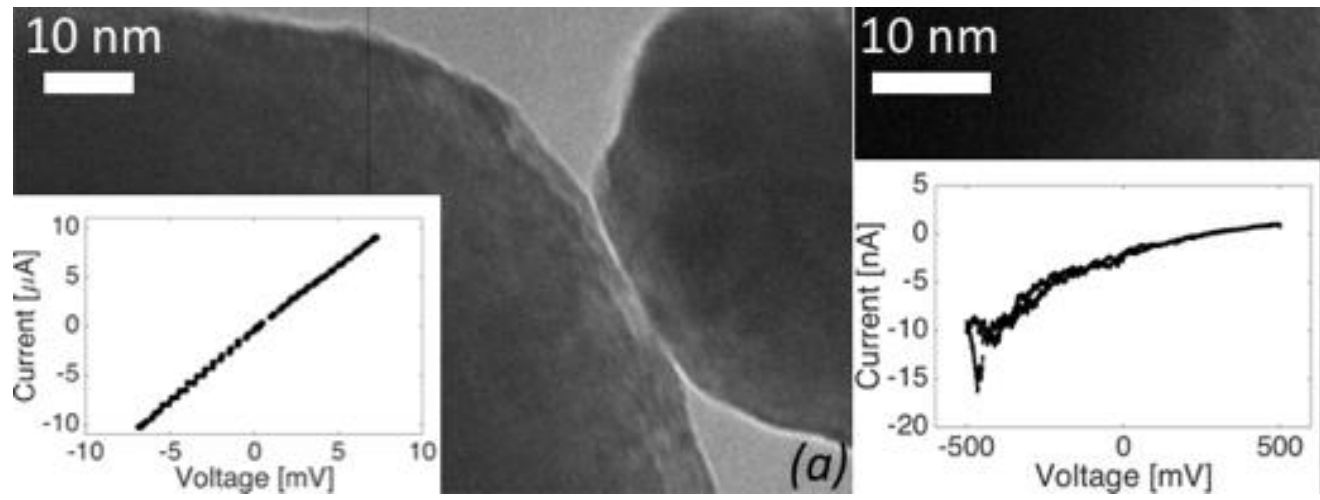

Figure 2. In-situ TEM electrical data was collected during TEM imaging of (a) a Pt/Ir tip contacting a W substrate and (b) a Si tip contacting a W substrate. The electrical measurements for each are shown in the insets. The direct imaging of the real-time apparent contact radius provides additional insights into the origin of the measured contact properties. 